\title{
Geometric embeddings of braid groups do not merge conjugacy classes
}

\author{
Juan González-Meneses*
}

July 16, 2013

\begin{abstract}
An embedding of the $m$-times punctured disc into the $n$-times punctured disc, for $n>m$, yields an embedding of the braid group on $m$ strands $B_{m}$ into the braid group on $n$ strands $B_{n}$, called a geometric embedding. The main example consists of adding $n-m$ trivial strands to the right of each braid on $m$ strands. We show that geometric embeddings do not merge conjugacy classes, meaning that if the images of two elements in $B_{m}$ by a geometric embedding are conjugate in $B_{n}$, the original elements are conjugate in $B_{m}$. We also show that the result does not hold, in general, for geometric embeddings of mapping class groups.
\end{abstract}

\section{Introduction}

Braid groups on $n$ strands $B_{n}$ were introduced by Artin [1] and can be defined in several different ways. The easiest one is by means of the following presentation, also due to Artin [2]

$$
B_{n}=\left\langle\begin{array}{l|ll}
\sigma_{1}, \ldots, \sigma_{n-1} & \begin{array}{ll}
\sigma_{i} \sigma_{j}=\sigma_{j} \sigma_{i} & |i-j|>1 \\
\sigma_{i} \sigma_{j} \sigma_{i}=\sigma_{j} \sigma_{i} \sigma_{j} & |i-j|=1
\end{array}
\end{array}\right\rangle
$$

If we see braids as homotopy classes of disjoint strands relative to their endpoints [3], the generator $\sigma_{i}$ corresponds to the crossing of strands in positions $i$ and $i+1$, in the positive sense (see Figure 1).

The group $B_{n}$ can also be seen as the mapping class group of the $n$-times punctured disc, relative to the boundary. More precisely, if $\mathbb{D}_{n}$ is a topological disc with $n$ punctures ( $n$ interior points removed), we can consider the set

*Partially supported by the Spanish research projects MTM2010-19355, P09-FQM-5112, FEDER and the Australian Research Councils Discovery Project DP1094072. 

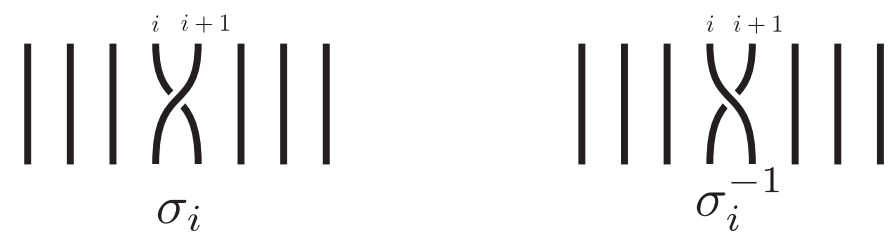

Figure 1: Classical (Artin) generators of $B_{n}$

Homeo $^{+}\left(\mathbb{D}_{n}, \partial \mathbb{D}_{n}\right)$ of orientation preserving homeomorphisms from $\mathbb{D}_{n}$ to itself fixing the boundary pointwise. Endowing $\mathrm{Homeo}^{+}\left(\mathbb{D}_{n}, \partial \mathbb{D}_{n}\right)$ with the usual compact-open topology, one defines the mapping class group $M C G\left(\mathbb{D}_{n}, \partial \mathbb{D}_{n}\right)=$ $\pi_{0}\left(\right.$ Homeo $\left.^{+}\left(\mathbb{D}_{n}, \partial \mathbb{D}_{n}\right)\right)$. That is, $M C G\left(\mathbb{D}_{n}, \partial \mathbb{D}_{n}\right)$ is the set of orientation preserving homeomorphisms of $\mathbb{D}_{n}$ (fixing the boundary pointwise), up to isotopy. It turns out that $B_{n} \simeq M C G\left(\mathbb{D}_{n}, \partial \mathbb{D}_{n}\right)$ ] .

There is a natural way to embed $B_{m}$ into $B_{n}$ for $m<n$ : Choose a topological embedding $\varphi: \mathbb{D}_{m} \rightarrow \mathbb{D}_{n}$ which sends punctures to punctures and $\partial \mathbb{D}_{m}$ to the interior of $\mathbb{D}_{n}$, and then extend every homeomorphism $f$ of $\mathbb{D}_{m}$ fixing the boundary pointwise to a homeomorphism $\varphi(f)$ of $\mathbb{D}_{n}$ which equals $\varphi \circ f \circ \varphi^{-1}$ in $\varphi\left(\mathbb{D}_{m}\right)$ and is the identity outside $\varphi\left(\mathbb{D}_{m}\right)$. This map which sends $f$ to $\varphi(f)$ is injective and respects isotopies, so it induces an embedding $\varphi: B_{m} \rightarrow B_{n}$, which is called a geometric embedding (see [14). Notice that we denoted three different maps (applied to points, to homeomorphisms and to mapping classes) with the same letter $\varphi$, hoping that this will not cause confusion.

The simplest example of a geometric embedding occurs when the disc embedding $\varphi$ is given by the inclusion, in the complex plane $\mathbb{C}$, of the $m$-times punctured disc of diameter $m-1 / 2$ whose punctures are the integers $1, \ldots, m$, into the $n$-times punctured disc of diameter $n$ whose punctures are the integers $1, \ldots, n$ (see Figure 2). We call this the standard embedding of $\mathbb{D}_{m}$ into $\mathbb{D}_{n}$, and will denote it by $\eta$. One can easily determine the standard geometric embedding $\eta: B_{m} \rightarrow B_{n}$ in terms of Artin's presentation, as in this case $\eta\left(\sigma_{i}\right)=\sigma_{i}$ for $i=1, \ldots, m-1$.

Actually, every geometric embedding $\varphi$ is conjugate to $\eta$, in the following sense. First, $\eta \circ \varphi^{-1}$ sends $\varphi\left(\mathbb{D}_{m}\right)$ to $\eta\left(\mathbb{D}_{m}\right)$. Then, as $\mathbb{D}_{n} \backslash \operatorname{int}\left(\varphi\left(\mathbb{D}_{m}\right)\right)$ and $\mathbb{D}_{n} \backslash \operatorname{int}\left(\eta\left(\mathbb{D}_{m}\right)\right)$ are both annuli with $n-m$ punctures, there is a homeomorphism from the former to the latter which coincides with $\eta \circ \varphi^{-1}$ on $\partial\left(\varphi\left(\mathbb{D}_{m}\right)\right)$. Gluing both homeomorphisms, we obtain an orientation preserving homeomorphism $g$ of $\mathbb{D}_{n}$ (fixing the boundary pointwise) such that $g \circ \varphi=\eta$, the standard embedding of $\mathbb{D}_{m}$ into $\mathbb{D}_{n}$. Considering $g$ as an element of $B_{n}$ we obtain

$$
\begin{aligned}
\eta: B_{m} & \longrightarrow B_{n} \\
f & \longmapsto g \circ \varphi(f) \circ g^{-1}
\end{aligned}
$$

as this is precisely the geometric embedding associated to the standard disc 


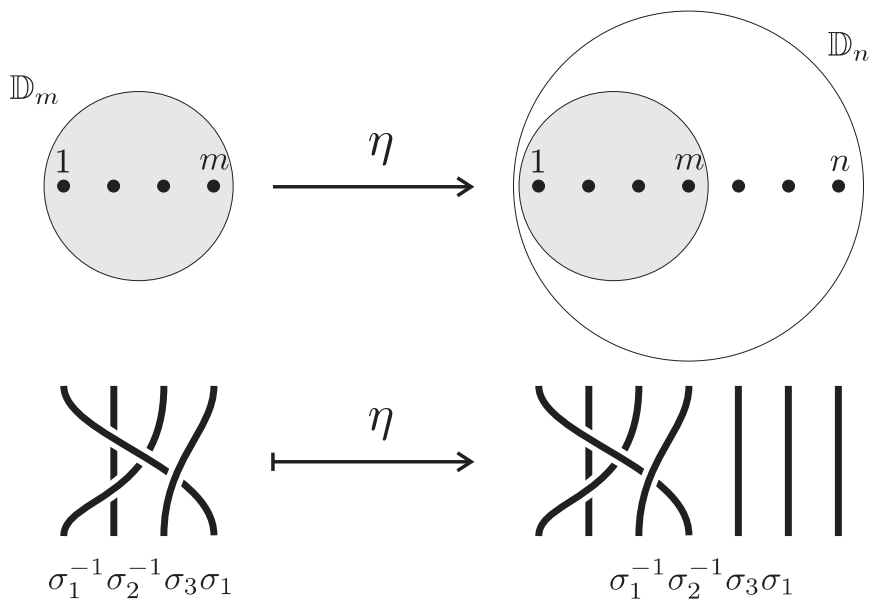

Figure 2: The standard geometric embedding of $B_{m}$ into $B_{n}$

embedding $g \circ \varphi=\eta: \mathbb{D}_{m} \rightarrow \mathbb{D}_{n}$.

Now the following question arises: Do geometric embeddings merge conjugacy classes of $B_{m}$ ? In other words, given a geometric embedding $\varphi: B_{m} \rightarrow B_{n}$, and given two non-conjugate elements $a, b \in B_{m}$, can $\varphi(a)$ and $\varphi(b)$ be conjugate in $B_{n}$ ?

In this paper, we answer the above question in the negative, by showing the following.

Theorem 1.1. Let $\eta: B_{m} \rightarrow B_{n}$ be the standard geometric embedding. For every $a, b \in B_{m}$, if $\eta(a)$ and $\eta(b)$ are conjugate in $B_{n}$, then a and $b$ are conjugate in $B_{m}$.

From the above result the following follows easily:

Corollary 1.2. Let $\varphi: B_{m} \rightarrow B_{n}$ be a geometric embedding. For every $a, b \in$ $B_{m}$, if $\varphi(a)$ and $\varphi(b)$ are conjugate in $B_{n}$, then $a$ and $b$ are conjugate in $B_{m}$.

Proof. Recall that $\eta$ is the geometric embedding corresponding to the disc embedding $g \circ \varphi$ for some homeomorphism $g$ of $\mathbb{D}_{n}$. If there exists $\alpha \in B_{n}$ such that $\varphi(b)=\alpha \varphi(a) \alpha^{-1}$, then $\eta(b)=g \varphi(b) g^{-1}=\left(g \alpha g^{-1}\right)\left(g \varphi(a) g^{-1}\right)\left(g \alpha^{-1} g^{-1}\right)=$ $\left(g \alpha g^{-1}\right) \eta(a)\left(g \alpha g^{-1}\right)^{-1}$. Thus $\eta(a)$ and $\eta(b)$ are conjugate, hence so are $a$ and $b$ by Theorem 1.1 .

In order to show Theorem 1.1, in Section 2 we will recall some basic facts from Nielsen-Thurston theory of braids and mapping classes, which will be used in Section 3 to prove the result. 

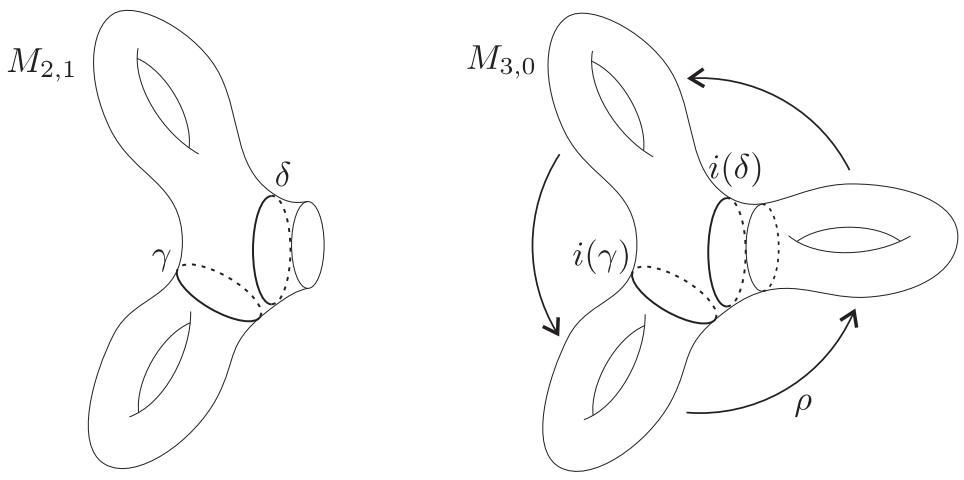

Figure 3: A geometric embedding which merges conjugacy classes

As several arguments in the proof of Theorem 1.1 use the general theory of mapping class groups, one is tempted to try to generalize the result to geometric embeddings of other mapping class groups. That is, if $M$ is a (possibly punctured) surface and $N$ is a subsurface such that $\partial N \cap \partial M=\emptyset$, the injection $i: N \hookrightarrow M$ induces a map $i_{*}: M C G(N, \partial N) \rightarrow M C G(M, \partial M)$ which in most cases is injective [12. Here is an example of such a geometric embedding which merges conjugacy classes.

Example 1.3. Let $M_{2,1}$ be the orientable surface of genus 2 with 1 boundary component, and let $M_{3,0}$ be the orientable closed surface of genus 3. Consider the geometric embedding $i: M_{2,1} \hookrightarrow M_{3,0}$ represented in Figure 3. The induced map $i_{*}: \operatorname{MCG}\left(M_{2,1}, \partial M_{2,1}\right) \rightarrow M C G\left(M_{3,0}\right)$ is injective as $\overline{M_{3,0} \backslash M_{2,1}}$ is neither a disc nor a punctured disc [12].

Let $\gamma$ and $\delta$ be two simple closed curves in $M_{2,0}$ as in Figure 3 , and let $\tau_{\gamma}$ and $\tau_{\delta}$ be their corresponding Dehn twists.

First we see that $\tau_{\gamma}$ is not conjugate to $\tau_{\delta}$ in $M C G\left(M_{2,1}, \partial M_{2,1}\right)$, as follows: Collapsing the boundary of $M_{2,1}$ to a puncture $p$ yields a group homomorphism from $M C G\left(M_{2,1}, \partial M_{2,1}\right)$ to $M C G\left(M_{2,0} \backslash\{p\}\right)$. The image of $\tau_{\delta}$ is trivial, while the image of $\tau_{\gamma}$ is not, hence these elements cannot be conjugate.

Now notice that the mapping class $\rho \in M C G\left(M_{3,0}\right)$ represented by a rotation of angle $2 \pi / 3$ sends the curve $i(\gamma)$ to the curve $i(\delta)$. This means that, in $M C G\left(M_{3,0}\right)$, one has $\rho \circ i_{*}\left(\tau_{\gamma}\right) \circ \rho^{-1}=i_{*}\left(\tau_{\delta}\right)$. Hence $i_{*}$ merges conjugacy classes. 


\section{Some facts from Nielsen-Thurston theory}

Recall that the braid group $B_{n}$ can be defined as $B_{n}=M C G\left(\mathbb{D}_{n}, \partial \mathbb{D}_{n}\right)$. There is a special braid, denoted $\Delta^{2}$, which corresponds to a Dehn twist along a curve which is parallel to the boundary of $\mathbb{D}_{n}$. This braid is called a full twist, or the Garside element of $B_{n}$, and it generates the center of $B_{n}$ [5].

If we quotient $B_{n}$ by its center, that is if we consider $B_{n} /\left\langle\Delta^{2}\right\rangle$, geometrically this corresponds to removing the condition that the boundary of $\mathbb{D}_{n}$ is fixed pointwise, so $B_{n} /\left\langle\Delta^{2}\right\rangle=M C G\left(\mathbb{D}_{n}\right)$. Notice that we could collapse the boundary of $\mathbb{D}_{n}$ to a new puncture, so this group becomes a subgroup of the mapping class group of the $(n+1)$-times punctured sphere.

This allows to study braids using the Nielsen-Thurston theory of mapping classes [13, 8, so braids can be classified into three geometric types:

- Periodic: If they have finite order modulo $\Delta^{2}$.

- Reducible, not periodic: If they are not periodic, and they preserve a family of disjoint, non-parallel simple closed curves in $\mathbb{D}_{n}$, each one enclosing more than 1 and less than $n$ punctures (such curves are called non-degenerate).

- pseudo-Anosov: If the induced mapping class of the $(n+1)$-times punctured sphere admits a representative which preserves two transverse measured foliations, scaling the measure of one of them by some real number $\lambda>0$, and the measure of the second one by $\lambda^{-1}$.

One can interpret this classification as follows: Periodic braids have a power which preserves all non-degenerate curves. Reducible (non-periodic) braids have a power which preserves some non-degenerate curves. Pseudo-Anosov braids have no power preserving a non-degenerate curve.

The most important case in this paper will be the second one: Reducible (but not periodic) braids. By definition, these braids preserve a family of non-degenerate curves. By [4], there is a distinguished family of curves associated to a reducible, non periodic braid $\beta$, called the canonical reduction system of $\beta$ and denoted $C R S(\beta)$.

The canonical reduction system $C R S(\beta)$ of a given braid $\beta$ (not necessarily reducible) is defined as follows. A simple closed curve $\mathcal{C}$ is said to be essential for $\beta$ if $\beta^{N}(\mathcal{C})=\mathcal{C}$ for some $N>0$, and if $\mathcal{C}^{\prime}$ is not fixed by any power of $\beta$ for every $\mathcal{C}^{\prime}$ having positive geometric intersection with $\mathcal{C}$ (that is, $\mathcal{C}^{\prime}$ cannot be isotoped to be disjoint from $\mathcal{C}$ ). Then $C R S(\beta)$ is the set of (isotopy classes of) essential curves for $\beta$. 
Some important facts concerning canonical reduction systems are the following [4:

- $C R S(\beta) \neq \emptyset$ if and only if $\beta$ is reducible, not periodic.

- $C R S\left(\alpha \circ \beta \circ \alpha^{-1}\right)=\alpha(C R S(\beta))$.

- $\mathbb{D}_{n} \backslash C R S(\beta)$ has several connected components. Given a power $\beta^{M}$ of $\beta$ which preserves each of these connected components (the permutation induced by $\beta^{M}$ on the set of connected components is trivial), the restriction of $\beta^{M}$ to each connected component is either periodic or pseudo-Anosov.

- $C R S(\beta)$ is the minimal family, under inclusion, of (isotopy classes of) non-degenerate curves satisfying the above property. In other words, a family of curves which satisfies the above property is $C R S(\beta)$ if and only if the family obtained by removing any of its curves does not satisfy the property.

One particular property of braids, compared to other mapping classes, is that cutting a punctured disc along disjoint non-degenerate curves yields again punctured discs (each curve plays the role of boundary of the outermost connected component it encloses). This implies that each reducible (non-periodic) braid $\beta \in B_{n}$ can be decomposed into simpler braids on fewer strands, cutting the disc along $C R S(\beta)$. Usually, in the theory of mapping classes, one takes a power $\beta^{M}$ such that each connected component is sent to itself, but in the case of braids this is not necessary, as one can see in [10.

In any case, if $\beta \in B_{n}$ is reducible and non-periodic, the outermost connected component of $\mathbb{D}_{n} \backslash C R S(\beta)$ is always preserved by $\beta$, and the restriction of $\beta$ to this component is a braid that we will call $\beta_{\text {ext }}$, the external component of $\beta$.

We point out that $\beta_{\text {ext }}$ is well defined up to conjugacy, as it depends on the way of collapsing the holes of the surface to punctures. Although there is a way to properly define the external and internal components of $\beta$ (see [10]), we shall not need it, as the only property of $\beta_{\text {ext }}$ we care about in this paper is whether it is trivial or not, and this is well defined up to conjugacy.

We will use the above results in the next section to show Theorem 1.1

\section{The standard geometric embedding does not merge conjugacy classes}

In this section we will show Theorem 1.1, Let $\eta: B_{m} \rightarrow B_{n}$ be the standard geometric embedding, and let $a, b \in B_{m}$ such that $\eta(a)$ and $\eta(b)$ are conjugate. 


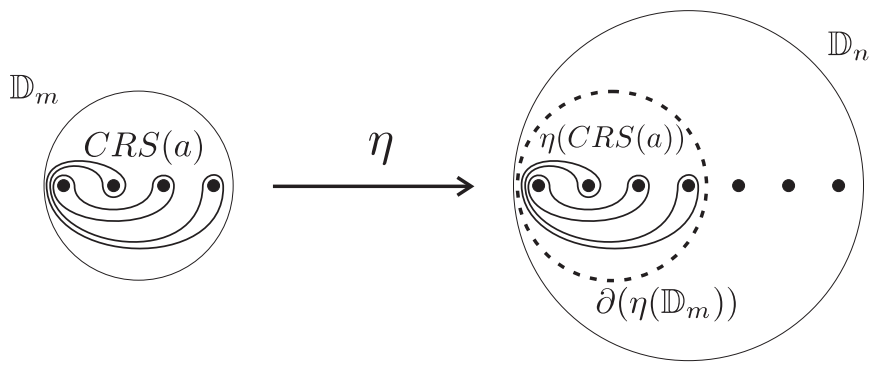

Figure 4: The canonical reduction system of $\eta(a)$ is obtained by applying $\eta$ to $C R S(a)$, and possibly adding $\partial\left(\eta\left(\mathbb{D}_{m}\right)\right)$.

If $a=1$ the result follows easily: one has $\eta(a)=1$ which implies that $\eta(b)=1$, and as $\eta$ is injective one obtains that $b=1$ too. We can then assume that both $a$ and $b$ are nontrivial.

First we must point out that $\eta(a)$ preserves the boundary of $\eta\left(\mathbb{D}_{m}\right)$, which is a non-degenerate curve of $\mathbb{D}_{n}$ hence $\eta(a)$ is not pseudo-Anosov. We shall now see that $\eta(a)$ cannot be periodic.

If $\eta(a)$ were periodic, there would be some integers $M, N$ with $N>0$ such that $\eta(a)^{N}=\Delta^{2 M}$. As $\Delta$ is a braid in which every strand crosses every other strand once in the positive sense, $\Delta^{2 M}$ is a braid in which every strand crosses every other strand, unless $M=0$. But in our case the $n$-th strand of $\eta(a)^{N}$ would not cross any other, so we deduce that $M=0$, hence $\eta(a)^{N}=1$. As braid groups have no torsion [7, Theorem 8] (see also [9, 6, 11]), it would follow that $\eta(a)=1$, so $a=1$, a contradiction. Therefore $\eta(a)$ is not periodic.

From the above arguments we obtain that $\eta(a)$ is reducible, non periodic. Hence $C R S(\eta(a)) \neq \emptyset$. We shall now compare $C R S(\eta(a))$ and $C R S(a)$, the latter being possibly empty, as $a$ can be either periodic, reducible or pseudo-Anosov.

Lemma 3.1. Under the above conditions, if $a_{\text {ext }}$ is trivial, then $C R S(\eta(a))=$ $\eta(C R S(a))$. Otherwise $C R S(\eta(a))=\eta(C R S(a)) \cup \partial\left(\eta\left(\mathbb{D}_{m}\right)\right)$. Moreover, in either case $\eta(a)_{\text {ext }}$ is trivial.

Proof. Recall that $\eta(a)$ is obtained by extending the homeomorphism $a$ of $\mathbb{D}_{m}$ by the identity to the whole $\mathbb{D}_{n}$, where $\mathbb{D}_{m}$ is embedded into $\mathbb{D}_{n}$ by the standard embedding $\eta$. This implies in particular that $\partial\left(\eta\left(\mathbb{D}_{m}\right)\right)$ is preserved by $\eta(a)$.

Since $C R S(a)$ is preserved by $a$, if we apply the standard disc embedding $\eta$ to this (possibly empty) family of curves, we obtain $\eta(C R S(a))$ which is preserved by $\eta(a)$. Hence $\Gamma=\eta(C R S(a)) \cup \partial\left(\eta\left(\mathbb{D}_{m}\right)\right)$ is a family of disjoint, simple, nonparallel, non-degenerate curves preserved by $\eta(a)$. Moreover, we claim that if $\eta(a)^{M}$ preserves the connected components of $\mathbb{D}_{n} \backslash \Gamma$, then $\eta(a)^{M}$ restricted to 
each connected component is either periodic or pseudo-Anosov. Indeed, $\eta(a)^{M}$ restricted to the outermost component of $\mathbb{D}_{n} \backslash \Gamma$ is the identity, while $\eta(a)^{M}$ restricted to any other component is equal to $a^{M}$ restricted to a component of $\mathbb{D}_{m} \backslash C R S(a)$, hence it is either periodic or pseudo-Anosov.

Therefore $C R S(\eta(a))$ is a subset of $\Gamma$, and we only need to see which curves of $\Gamma$ can be removed so that the restriction of $\eta(a)^{M}$ to each connected component is still either periodic or pseudo-Anosov.

Given $\mathcal{C} \in \eta(C R S(a))$, removing $\mathcal{C}$ from $\Gamma$ merges two connected components. That is, if we consider $\Gamma^{\prime}=\Gamma \backslash\{\mathcal{C}\}$, there is a connected component of $\mathbb{D}_{n} \backslash \Gamma^{\prime}$ which is the union of $\mathcal{C}$ and of two connected components of $\mathbb{D}_{n} \backslash \Gamma$. If the restriction of $\eta(a)^{M}$ to this component were periodic or pseudo-Anosov, this would imply that $\eta^{-1}(\mathcal{C})$ can be removed from $C R S(a)$, which is not true. Hence all curves of $\eta(C R S(a))$ belong to $C R S(\eta(a))$, so the only curve which could possibly be removed from $\Gamma$ to obtain $C R S(\eta(a))$ is $\partial\left(\eta\left(\mathbb{D}_{m}\right)\right)$.

Now suppose that $a_{\text {ext }}$ is trivial. Then the restriction of $\eta(a)$ (and of every power of $\eta(a))$ to the outermost component of $\mathbb{D}_{n} \backslash \eta(C R S(a))$ is also trivial. Hence the restrictions of $\eta(a)^{M}$ to the connected components of $\mathbb{D}_{n} \backslash \eta(C R S(a))$ are either periodic or pseudo-Anosov, therefore $C R S(\eta(a))=\eta(C R S(a))$.

Finally, suppose that $a_{\text {ext }}$ is not trivial. Let $S$ be the outermost component of $\mathbb{D}_{n} \backslash \eta(C R S(a))$, and consider $\eta(a)^{M}$ restricted to $S$. This mapping class is still a braid, as $S$ is (homeomorphic to) a punctured disc. Notice that $\left(\eta(a)^{M}\right)_{\left.\right|_{S}}$ is not periodic: as the last puncture of $\mathbb{D}_{n}$ does not cross any other, if it were periodic it should be trivial, but this is not the case. Also, $\left(\eta(a)^{M}\right)_{\left.\right|_{S}}$ is not pseudo-Anosov, as it preserves the non-degenerate curve $\partial\left(\eta\left(\mathbb{D}_{m}\right)\right)$. Hence we cannot remove $\partial\left(\eta\left(\mathbb{D}_{m}\right)\right)$ from $\Gamma$ in this case, and therefore $C R S(\eta(a))=\Gamma$.

In both cases the restriction of $\eta(a)$ to the outermost component of $\mathbb{D}_{n} \backslash C R S(\eta(a))$ is trivial, that is $\eta(a)_{e x t}$ is trivial. This ends the proof of Lemma 3.1

Now suppose that $\eta(a)$ and $\eta(b)$ are conjugate in $B_{n}$, and let $\alpha \in B_{n}$ such that $\alpha \eta(a) \alpha^{-1}=\eta(b)$. Then we have $C R S(\eta(b))=\alpha(C R S(\eta(a)))$.

By Lemma 3.1. $C R S(\eta(a))$ has a curve enclosing $m$ punctures if and only if $a_{\text {ext }}$ is trivial. Therefore $a_{\text {ext }}$ is trivial if and only if so is $b_{\text {ext }}$.

Let $C R S_{\text {out }}(\eta(a))$ be the set of outermost curves in $C R S(\eta(a))$, and define $C R S_{\text {out }}(\eta(b))$ similarly. As nested curves preserve their relative position under the homeomorphism $\alpha$, it follows that $C R S_{\text {out }}(\eta(b))=\alpha\left(C R S_{\text {out }}(\eta(a))\right)$.

Let us denote by $S_{a}$ the outermost connected component of $\mathbb{D}_{n} \backslash C R S(\eta(a))$ and by $S_{b}$ the outermost connected component of $\mathbb{D}_{n} \backslash C R S(\eta(b))$. As $\alpha$ sends $\mathcal{C} R S_{\text {out }}(\eta(a))$ to $\mathcal{C} R S_{\text {out }}(\eta(b))$, we have $\alpha\left(S_{a}\right)=S_{b}$.

Suppose that $a_{\text {ext }}$ (and thus $b_{\text {ext }}$ ) is nontrivial. In this case $S_{a}=S_{b}=\mathbb{D}_{n} \backslash \eta\left(\mathbb{D}_{m}\right)$ 
so $\alpha$ preserves $\eta\left(\mathbb{D}_{m}\right)$, and we can clearly take a representative of $\alpha$ which fixes $\partial\left(\eta\left(\mathbb{D}_{m}\right)\right)$ pointwise. Let $\alpha^{\prime}$ be the restriction of this representative to $\eta\left(\mathbb{D}_{m}\right)$, and let $\alpha_{0}=\eta^{-1}\left(\alpha^{\prime}\right) \in B_{m}$.

Let us see that in this case $b=\alpha_{0} a \alpha_{0}^{-1}$. Indeed,

$$
\begin{gathered}
b=\eta^{-1}\left(\eta(b)_{\left.\left.\right|_{\eta(\mathbb{D} m}\right)}\right)=\eta^{-1}\left(\left(\alpha \eta(a) \alpha^{-1}\right)_{\left.\left.\right|_{\eta(\mathbb{D} m)}\right)}\right)= \\
=\eta^{-1}\left(\alpha^{\prime} \eta(a)_{\left.\right|_{\eta(\mathbb{D} m)}}\left(\alpha^{\prime}\right)^{-1}\right)=\alpha_{0} \eta^{-1}\left(\eta(a)_{\left.\right|_{\eta\left(\mathbb{D}_{m}\right)}}\right) \alpha_{0}^{-1}=\alpha_{0} a \alpha_{0}^{-1} .
\end{gathered}
$$

Now suppose that $a_{\text {ext }}$ (and thus $b_{\text {ext }}$ ) is trivial. This implies by Lemma 3.1 that all curves in $C R S(\eta(a))$ and $C R S(\eta(b))$ belong to the interior of $\eta\left(\mathbb{D}_{m}\right)$. Hence $\partial\left(\eta\left(\mathbb{D}_{m}\right)\right)$ belongs to the interior of both $S_{a}$ and $S_{b}$. Let $\mathcal{C}_{1}=\partial\left(\eta\left(\mathbb{D}_{m}\right)\right)$ and $\mathcal{C}_{2}=\alpha\left(\mathcal{C}_{1}\right)$. Recall that $S_{b}$ is isomorphic to a punctured disc, and that $\mathcal{C}_{1}$ and $\mathcal{C}_{2}$ are interior closed simple curves of $S_{b}$. Moreover, as the annulus determined by $\mathcal{C}_{1}$ (resp. $\mathcal{C}_{2}$ ) and $\partial \mathbb{D}_{n}$ contains exactly $n-m$ punctures, there is a homeomorphism of $S_{b} \cup C R S_{\text {out }}(\eta(b))$ which sends $\mathcal{C}_{2}$ to $\mathcal{C}_{1}$, and leaves $C R S_{\text {out }}(\eta(b))$ and $\partial \mathbb{D}_{n}$ fixed pointwise. Extending this homeomorphism by the identity to the whole $\mathbb{D}_{n}$, we obtain a homeomorphism $\beta$ of $\mathbb{D}_{n}$ with support in $S_{b}$, such that $\beta\left(\mathcal{C}_{2}\right)=\mathcal{C}_{1}$.

If we consider $\gamma=\beta \circ \alpha$, we notice that $\gamma\left(\mathcal{C}_{1}\right)=\beta\left(\alpha\left(\mathcal{C}_{1}\right)\right)=\beta\left(\mathcal{C}_{2}\right)=\mathcal{C}_{1}$. That is $\gamma\left(\partial\left(\eta\left(\mathbb{D}_{m}\right)\right)\right)=\partial\left(\eta\left(\mathbb{D}_{m}\right)\right)$. Hence $\gamma\left(\eta\left(\mathbb{D}_{m}\right)\right)=\eta\left(\mathbb{D}_{m}\right)$, and we can take a representative of $\gamma$ which fixes $\partial\left(\eta\left(\mathbb{D}_{m}\right)\right)$ pointwise. Moreover, $\beta$ and $\eta(b)$ have disjoint support, hence they commute. Therefore:

$$
\gamma \eta(a) \gamma^{-1}=\beta \alpha \eta(a) \alpha^{-1} \beta^{-1}=\beta \eta(b) \beta^{-1}=\eta(b) .
$$

We then have a braid $\gamma \in B_{n}$ which conjugates $\eta(a)$ to $\eta(b)$ and preserves $\eta\left(\mathbb{D}_{m}\right)$. Denoting $\gamma^{\prime}=\gamma_{\left.\left.\right|_{\eta(\mathbb{D}}\right)}$, and $\gamma_{0}=\eta^{-1}\left(\gamma^{\prime}\right) \in B_{m}$, it follows as above that $\gamma_{0} a \gamma_{0}^{-1}=b$.

This shows that in every case, $a$ and $b$ are conjugate in $B_{m}$, finishing the proof of Theorem 1.1.

Acknowledgements: It is a pleasure for me to write this paper at the occasion of the 70th birthday of Professor González-Acuña. The question we address in this paper was raised to me by Patrick Dehornoy, and some time later by Ivan Marin. I thank both of them, and I specially thank Ivan Marin for encouraging me to write this paper. I also thank Bert Wiest for some interesting comments, and for sending me an alternative proof of the main result using just properties of braid strands. Part of this work was done during a stay at the Centre de Recerca Matemàtica (CRM) in Bellaterra (Barcelona, Spain), to which I thank for its hospitality. 


\section{References}

[1] E. Artin. Theorie der Zöpfe. Abh. Math. Sem. Univ. Hamburg 4 (1925), no. $1,47-72$.

[2] E. Artin. Theory of braids. Ann. of Math. 48 (1947), no. 2, 101-126.

[3] J. S. Birman. Braids, links, and mapping class groups. Annals of Mathematics Studies, No. 82. Princeton University Press, 1974.

[4] J. S. Birman, A. Lubotzky, J. McCarthy, Abelian and solvable subgroups of the mapping class groups. Duke Math. J. 50 (1983), no. 4, 1107-1120.

[5] W.-L. Chow. On the algebraical braid group. Ann. of Math. (2) 49, (1948). 654-658.

[6] J. L. Dyer. The algebraic braid groups are torsion-free: An algebraic proof. Math. Z. 172 (1980), no. 2, 157-160.

[7] E. Fadell, L. Neuwirth. Configuration spaces. Math. Scand. 101962 111-118.

[8] A. Fathi, F. Laudenbach, V. Poénaru. Travaux de Thurston sur les surfaces. Astérisque No. 66-67 (1991). SMF, Paris, 1991.

[9] R. Fox, L. Neuwirth. The braid groups. Math. Scand. 101962 119-126.

[10] J. González-Meneses. On reduction curves and Garside properties of braids. Contemporary Mathematics 538 (2011), 227-244.

[11] J. González-Meneses. Basic results on braid groups. Ann. Math. Blaise Pascal 18, no. 1 (2011), 15-59.

[12] L. Paris, D. Rolfsen. Geometric subgroups of mapping class groups. J. reine angew. Math. 521 (2000), 4783.

[13] W. P. Thurston. On the geometry and dynamics of diffeomorphisms of surfaces. Bull. Amer. Math. Soc. (N.S.) 19 (1988), no. 2, 417-431.

[14] B. Wajnryb. Artin groups and geometric monodromy. Invent. Math. 138 (1999), no. 3, 563-571.

Juan González-Meneses

Departamento de Álgebra

Facultad de Matemáticas

Instituto de Matemáticas (IMUS)

Universidad de Sevilla

Apdo. 1160

41080 Sevilla (Spain)

meneses@us.es 